\title{
Nitroglycerin enhances proliferation and osteoblastic differentiation in human mesenchymal stem cells via nitric oxide pathway ${ }^{1}$
}

\author{
Li HUANG ${ }^{2}$, Ni QIU², Che ZHANG ${ }^{2,4}$, Hong-yan $\mathrm{WEI}^{2}$, Ya-lin $\mathrm{LI}^{2}$, Hong-hao ZHOU ${ }^{2}$, Zhou-sheng XIAO ${ }^{2,3,5}$ \\ ${ }^{2}$ Institute of Clinical Pharmacology, Central South University, Changsha 410078, China; ${ }^{3}$ The Kidney Institute, University of Kansas Medical Center, \\ Kansas City, Kansas 66160, USA; ${ }^{4}$ Department of Pharmacy, Taihe Hospital Affiliated to Yunyang Medical College, Shiyan 442000, China
}

\section{Key words}

nitroglycerin; human bone marrow-derived mesenchymal stem cells; nitric oxide

\begin{abstract}
${ }^{1}$ This study was supported by grants from the Teaching and Research Award Program for Outstanding Young Teachers (TRAPOYT) in Higher Education Institutions of MOE, China (No 30040002); the National Natural Science Foundation of China (No 30171085 ); and the National Institutes of Health, USA (No RO1-AR049712).

${ }^{5}$ Correspondence to Prof Zhou-sheng XIAO.

Phn 1-913-588-0703.

Fax 1-913-588-9251.

E-mailzxiao@kumc.edu
\end{abstract}

Received 2007-06-28

Accepted 2008-01-16

doi: $10.1111 / \mathrm{j} .1745-7254.2008 .00778 . x$

\begin{abstract}
Aim: To investigate the effect of nitroglycerin (NTG) on cell proliferation and osteoblastic differentiation of human bone marrow-derived mesenchymal stem cells (HBMSC) and its mechanisms. Methods: Primary HBMSC were cultured in osteogenic differentiation medium consisting of phenol red-free $\alpha$-minimum essential media plus $10 \%$ fetal bovine serum (dextran-coated charcoal stripped) supplemented with $10 \mathrm{nmol} / \mathrm{L}$ dexamethasone, $50 \mathrm{mg} / \mathrm{L}$ ascorbic acid, and $10 \mathrm{mmol} / \mathrm{L}$ $\beta$-glycerophosphate for inducing osteoblastic differentiation. The cells were treated with NTG $(0.1-10 \mu \mathrm{mol} / \mathrm{L})$ alone or concurrent incubation with different nitric oxide synthase (NOS) inhibitors. Nitric oxide (NO) production was measured by using a commercial NO kit. Cell proliferation was measured by 5-bromodeoxyuridine (BrdU) incorporation. The osteoblastic differentiation of HBMSC culture was evaluated by measuring cellular alkaline phosphatase (ALP) activity and calcium deposition, as well as osteoblastic markers by real-time RT-PCR. Results: The treatment of HBMSC with NTG $(0.1-10 \mu \mathrm{mol} / \mathrm{L})$ led to a dose-dependent increase of NO production in the conditional medium. The release of NO by NTG resulted in increased cell proliferation and osteoblastic differentiation of HBMSC, as evidenced by the increment of the BrdU incorporation, the induction of ALP activity in the early stage, and the calcium deposition in the latter stage. The increment of NO production was also correlated with the upregulation of osteoblastic markers in HBMSC cultures. However, the stimulatory effect of NTG $(10 \mu \mathrm{mol} / \mathrm{L})$ could not be abolished by either $N^{\mathrm{G}}$-nitro- $L$-arginine methyl ester, an antagonist of endothelial NOS, or 1400W, a selective blocker of inducible NOS activity. Conclusion: NTG stimulates cell proliferation and osteoblastic differentiation of HBMSC through a direct release of NO, which is independent on intracellular NOS activity.
\end{abstract}

\section{Introduction}

Osteoporosis is a common and metabolic bone disease in older women, whose features include severe osteopenia and the cataplasias of bone microarchitecture, which increase the friability and risk of bone fracture. Estrogen $\left(\mathrm{E}_{2}\right)$ replacement therapy used to be the primary treatment option for post-menopausal women to reverse the osteopenia caused by osteoporosis, but its side-effects, such as increasing the risk of breast cancer and endometrial cancer, limit its application $^{[1]}$.

It has been reported that nitric oxide (NO) is a mechanism of $\mathrm{E}_{2}$ treatment for osteoporosis ${ }^{[2]}$. It has also been confirmed that NO plays an important role in the course of osteoporosis, which is supported by the fact that bone formation is blocked in NO synthase (NOS)-knockout mice ${ }^{[3]}$. Therefore, nitroglycerin (NTG), a common NO donor, might be a better choice for osteoporosis therapy, and the benefits of the NO donor to bone formation shed new light on the treatment for osteoporosis. NTG has been frequently used for cardiovascular disease in clinics for over a century, the security of which has been generally accepted at the routine dosage. Nowadays, it is still a hot topic since its metabolic 
product NO exerts numerous functions during message transduction in many systems. Several researches have demonstrated that NTG could restore the bone mineral density(BMD) in animal osteoporosis models ${ }^{[4]}$ and oophorectomy-induced bone loss in women ${ }^{[5]}$ as efficient as $\mathrm{E}_{2}$ treatment. In addition to the medication, osseous tissue engineering sheds new light on the treatments of osteoporosis. Bone marrow-derived mesenchymal stem cells (BMSC), which are derived from the seed cells of tissue engineering and are of potency for multidirectional differentiation, have been the focus of study. BMSC can be extracted from humans and several animals in the bone marrow. They differentiate towards osteoblasts in the mineralized culture, thus, have the ability to heal fractures and repair bone defects in osteoporosis in osseous tissue engineering ${ }^{[6,7]}$. We performed further investigations at the cellular level to detect the effects and mechanism of NTG on human BMSC cultures.

\section{Materials and methods}

Reagents $\alpha$-Minimum essential media ( $\alpha$-MEM), phenol red-free $\alpha$-MEM, fetal bovine serum (FBS), penicillin-streptomycin solution, and SDS were obtained from GIBCO BRL (Grand Island, NY, USA). Trypsin (1:125), ascorbic acid (Vitamin C), $\beta$-glycerophosphate $(\beta$-GP), dexamethasone (DXM), $p$-nitrophenyl phosphate ( $p$-NPP), $p$-nitrophenol, diethanolamine, DMSO, and dextran-coated charcoal were purchased from Sigma (St Louis, MO, USA). Tetrazolium was purchased from Sino-American Biotechnology (USA). Nitroglycerin was purchased from YiMin (Beijing, China). $N^{\mathrm{G}}$-nitro- $L$-argininemethyl ester, $(L-\mathrm{NAME}), N$-(3-[aminomethyl] benzyl) acetamidine (dihydrochloride, $1400 \mathrm{~W}$ ), the Bradford protein assay kit, and the BCA protein assay kit were purchased from Beyotime Institute of Biotechnology (Haimen, China). The nitrate/nitrite colorimetric assay kit was purchased from Jingmei Biotechnology (Beijing, China). The calcium assay kit was obtained from Kehua Bio-engineering (Shanghai, China). The 5-bromodeoxyuridine (BrdU) cell proliferation assay kit was purchased from Chemicon and Millipore (Billerica, MA, USA). All other chemicals were of analytical grade and were purchased from Shanghai Biotechnology (Shanghai, China). Tissue culture plastic wares were purchased from Corning-Costar (Corning-Costar, NY, USA); iQ SYBR green supermix was obtained from Bio-Rad Laboratories (Hercules, CA, USA).

Experimental equipment A carbon dioxide $\left(\mathrm{CO}_{2}\right)$ incubator was purchased from Sheldon Manufacturing (Cornelius, USA). A super clean bench was obtained from Jiangsu Purification Group (Suzhou, China). An inverted microscope was purchased from Nikon (Tokyo, Japan). Electronic balance and super-speed refrigerated centrifuge were purchased from Sigma (USA). An ultrasonic cell disrupter and UV-visible spectrophotometer were purchased from Shimadzu (Kyoto, Japan). A Multiskan ascent microplate reader was purchased from Thermo Labsystems (Helsinki, Finland) and the iCycler was purchased from Bio-Rad (USA).

Cell culture and treatment The human BMSC (HBMSC) were immortalized with SV40 large and small T-antigens from primary HBMSC cultures, as previously described ${ }^{[8,9]}$. This study was approved by the Ethics Committee Board of Xiangya School of Medicine, Central South University, Hunan, China. Briefly, the cells were maintained in $\alpha$-MEM supplemented with $15 \%$ FBS in a humidified incubator with $5 \% \mathrm{CO}_{2}$ and $95 \%$ air at a temperature of $37{ }^{\circ} \mathrm{C}$. After sub-confluence, the cells were plated overnight into either 96 -well or 24-well plates at the indicated density. The cells in the 24-well plates were switched to the differentiation media: phenol red-free $\alpha$-MEM containing 10\% FBS (dextran-coated charcoal stripped), 10 $\mathrm{nmol} / \mathrm{LDXM}, 50 \mathrm{mg} / \mathrm{L}$ vitamin $\mathrm{C}$, and $10 \mathrm{mmol} / \mathrm{L} \beta$-GP for inducing osteoblastic differentiation of HBMSC. In the subsequent experiments, the first day of culture in the differentiation media was defined as $\mathrm{d} 0$, and the cell treatments were added on $\mathrm{d} 0$. For the treatments, the culture media were replaced every $2 \mathrm{~d}$ up to the indicated days for harvesting.

Cytotoxicity assay The cytotoxicity of NTG to the cells was carried out using methyl thiazolyl tetrazolium (MTT) chronometry assay. The HBMSC cells were digested with $0.25 \%$ trypsin before cell counting, and then plated into a 96-well plate at a density of $1 \times 10^{4}$ cells $/ \mathrm{mL} ; 0.2 \mathrm{~mL}$ cell suspension was added to each well. The cells were supplied with $\alpha$ MEM containing $10 \%$ FBS for $24 \mathrm{~h}$ to achieve $60 \%$ confluence after adherence. Then the media were replaced and the cells were treated with different concentrations of NTG for $72 \mathrm{~h}$. NTG was dissolved in less than $0.1 \%$ ethanol, which served as the vehicle control. After $72 \mathrm{~h}$, the cells were incubated with MTT [dissolved in $5 \mathrm{~g} / \mathrm{L}$ phosphatebuffered saline (PBS)] for an additional $4 \mathrm{~h}$, then the media were removed and $200 \mu \mathrm{L}$ DMSO was added to each well for the following assays. The plates were shaken for $10 \mathrm{~min}$ or incubated at $37^{\circ} \mathrm{C}$ for 15 min to obtain a sufficient extraction of the MTT products, then optical density $(O D)$ at $490 \mathrm{~nm}$ was detected with a 96-well microplate reader. There was a direct correlation between the $O D$ value and the concentration of blue products, which represented the number of live cells ${ }^{[10]}$, and was expressed as the percentage of the control group in this study.

Measurement of NO production NO production was measured as the total content of nitrate and nitrite in the 
culture media. A nitrite colorimetric assay kit was employed using the nitrate reductase method, as described before ${ }^{[11]}$. Absorbance was then measured at $530 \mathrm{~nm}$ and the NO content in the culture media was expressed as the nitrite concentration, which was determined by comparing with the $\mathrm{KNO}_{3}$ standard. The NO concentration was expressed as $\mu \mathrm{mol} / \mathrm{L}$.

BrdU cell proliferation assay Measurements were performed as per the instructions provided in the kit. The cells were plated at $2 \times 10^{5}$ cells $/ \mathrm{mL}$ in $100 \mu \mathrm{L} \alpha$-MEM containing $10 \%$ FBS in each well. After adherence, the media were replaced with $\alpha$-MEM containing $0.1 \%$ FBS for another $24 \mathrm{~h}$ to achieve the synchronization of cell growth. Then the cells were treated with various concentrations of NTG plus BrdU in $\alpha$-MEM containing $7.5 \%$ FBS for an additional $24 \mathrm{~h}$. At the end of the BrdU labeling, the media were removed, and the plate was dried for $5 \mathrm{~min}$. The fixing solution was then added at room temperature for $30 \mathrm{~min}$. The plate was washed again with wash buffer for $3 \times 5 \mathrm{~min}$, then the following reagents were added in order: anti-BrdU, goat antimouse immunoglobulin G POD, and TMB by repeating the incubation, washing, and drying procedures according to the manufacturer's protocol. The $O D$ value was detected at $450 \mathrm{~nm}$ after the acid stop solution was added. The group in which cell proliferation was most active showed a higher $O D$ value, which was expressed as the percentage of the control group in this study.

Alkaline phosphatase activity Cellular alkaline phosphatase (ALP) activity was assayed according to our previous study ${ }^{[12]}$. At the end of the $8 \mathrm{~d}$ treatments, the cells were harvested; the media was used for the nitrite production assay. The cells were washed twice with PBS and treated with $0.25 \%$ trypsin for $10 \mathrm{~min}$ to allow for detachment, and then collected for the enzyme activity test. Cellular ALP activity was determined using $p$-NPP $(90 \mathrm{mmol} / \mathrm{L})$ as the substrate (pH 10.3) at $37{ }^{\circ} \mathrm{C}$ for $30 \mathrm{~min}$. The $O D$ value was then read at $405 \mathrm{~nm}$, and the total protein content was measured with the Bradford protein assay kit. ALP activity was expressed as nmol $\cdot \mathrm{min}^{-1} \cdot \mu \mathrm{g}^{-1}$ protein.

Quantitation of calcium deposition The calcification of HBMSC was assessed by modification of the Wada procedure $^{[12]}$. After $12 \mathrm{~d}$ co-incubation with the reagents, the cells were washed twice with PBS and then decalcified with 0.6 $\mathrm{mol} / \mathrm{L} \mathrm{HCl}$ for $24 \mathrm{~h}$. The calcium content was determined by measuring the calcium concentration in the $\mathrm{HCl}$ supernatant by the methylthymol blue method using the calcium assay kit. The cells were washed with PBS and then underwent schizolysis with the commixture of $0.1 \mathrm{~mol} / \mathrm{L} \mathrm{NaOH}$, and $0.1 \%$ SDS. The total protein content was detected with a BCA protein assay kit to normalize the calcium content, which was expressed as $\mu \mathrm{g} \cdot \mu \mathrm{g}^{-1}$ protein.

Real-time RT-PCR For the quantitative real-time RTPCR, $2.0 \mu \mathrm{g}$ total RNA isolated from HBMSC cultures was reverse transcribed, as previously described ${ }^{[22]}$. The PCR reactions contained $100 \mathrm{ng}$ template (cDNA or RNA), 300 nmol each of forward and reverse primers, and $1 \times$ iQ SYBR green supermix in $50 \mu \mathrm{L}$. The threshold cycle $(\mathrm{Ct})$ of the tested gene product from the indicated group was normalized to the $\mathrm{Ct}$ for $\mathrm{GAPDH}^{[8]}$.

Statistical analysis The parameters were expressed as mean \pm SD. Statistical significance was evaluated by oneway ANOVA assay, and a $P$-value of less than 0.05 was considered statistically significant.

\section{Results}

Cytotoxicity of NTG on HBMSC To investigate the cytotoxicity of NTG on HBMSC, the cells were treated with a broad dose of NTG from $1 \mathrm{nmol} / \mathrm{L}$ to $1 \mathrm{mmol} / \mathrm{L}$, as mentioned in Materials and methods. When NTG reached $0.1-1 \mathrm{mmol} / \mathrm{L}$, the cells floated in the media after $24 \mathrm{~h}$ treatment, which indicated the notable cytotoxicity of NTG under $0.1-1 \mathrm{mmol} / \mathrm{L}$ concentrations. Otherwise, there was no obvious cytotoxicity from $1 \mathrm{nmol} / \mathrm{L}$ to $10 \mu \mathrm{mol} / \mathrm{L}$, as compared with the control group. In addition, there was a greater amount of cells observed in the 1 - and $10 \mu \mathrm{mol} / \mathrm{L}$-treated groups, indicated NTG at $1 \mu \mathrm{mol} / \mathrm{L}$ and $10 \mu \mathrm{mol} / \mathrm{L}$ promoted cell proliferation in HBMSC cultures (Figure 1).

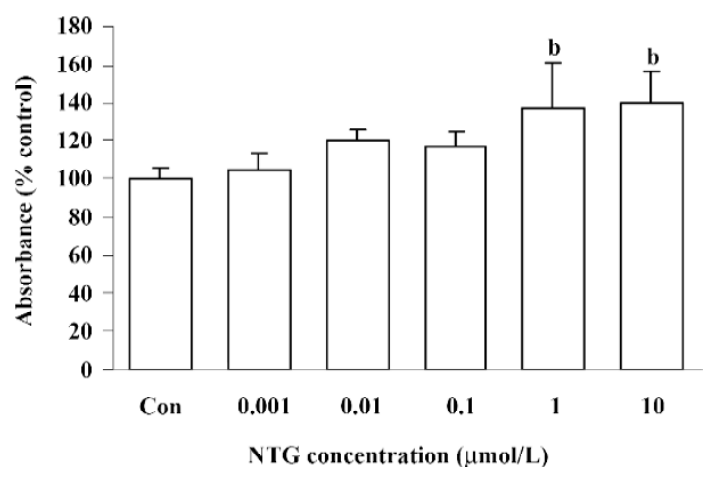

Figure 1. Cytotoxicity of NTG on HBMSC. MTT OD $(\lambda / \mathrm{nm}=490)$ value was measured and expressed as the percentage of the control. No obvious cytotoxicity was observed at 0.001-10 $\mu \mathrm{mol} / \mathrm{L}$ NTG. When NTG reached 1 and $10 \mu \mathrm{mol} / \mathrm{L}$, the cell number was significantly increased, reflecting an increase in cell proliferation. $n=3$. Mean \pm SD. ${ }^{\mathrm{b}} P<0.05$ vs vehicle control.

Increased NO production in NTG-treated HBMSC cultures NTG $(0.01-10 \mu \mathrm{mol} / \mathrm{L})$ treatment resulted in a dosedependent increase of NO production in the conditioned 
media on d 4 (Figure 2), d 8 (Figure 5A), and d 12 (Figure 6A). As shown in Figure 2, $0.1 \mu \mathrm{mol} / \mathrm{L}$ NTG on $\mathrm{d} 4 \mathrm{had}$ a significant increase on NO production compared to the control; the maximal outcome of NO production was $10 \mu \mathrm{mol} / \mathrm{L}$. To examine the role of NOS on NO production, $L$-NAME [a selective inhibitor of endothelial NOS (eNOS)] and 1400W [a selective inhibitor of inducible NOS (iNOS)] were employed in this study. As shown in Figure 3, $10 \mu \mathrm{mol} / \mathrm{L}$ NTG significantly increased the NO outcome in the conditioned media, however, there was no significant change observed in NO production between the NTG-treated group and the NTG+LNAME $(300 \mu \mathrm{mol} / \mathrm{L})$ or $1400 \mathrm{~W}(100 \mu \mathrm{mol} / \mathrm{L})$ groups, indicating the increment of NO production was independent of either eNOS or iNOS activity in HBMSC cultures. L-NAME or $1400 \mathrm{~W}$ alone had no obvious effect on NO production in HBMSC cultures.

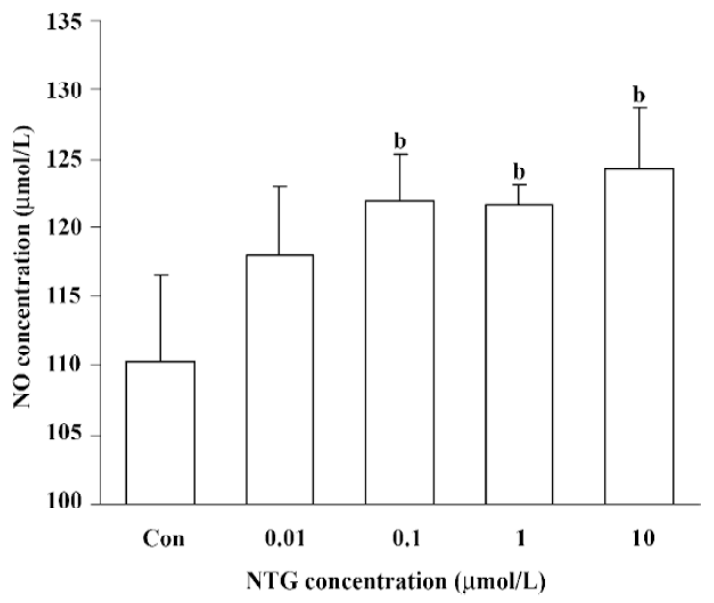

Figure 2. Effect of NTG on NO production in HBMSC cultures. HBMSC were treated with NTG at the indicated concentrations. NO production was increased in a dose-dependent manner. $n=3$. Mean \pm SD. ${ }^{\mathrm{b}} P<0.05 \mathrm{vs}$ vehicle control.

\section{Effect of NTG on cell proliferation of HBMSC cultures} As shown in Figure 4, NTG $(0.1-10 \mu \mathrm{mol} / \mathrm{L})$ dose dependently increased the BrdU incorporation into HBMSC cultures, indicating that NTG enhanced cell DNA synthesis and the cell growth rate. Compared to the increased maximal NO production, the maximal effect of NTG on cell growth was $1 \mu \mathrm{mol} / \mathrm{L}$, but 5 or $10 \mu \mathrm{mol} / \mathrm{L}$ NTG showed a slight reduction on cell proliferation, suggesting that $1 \mu \mathrm{mol} / \mathrm{L} \mathrm{NTG}$ could be a transition point for cell proliferation in HBMSC cultures.

Effect of NTG on osteoblastic differentiation of HBMSC cultures According to our previous study, d 8 of ALP activity and d 12 of calcium deposition were used to evaluate osteoblastic differentiation in HBMSC cultures ${ }^{[8]}$. In the

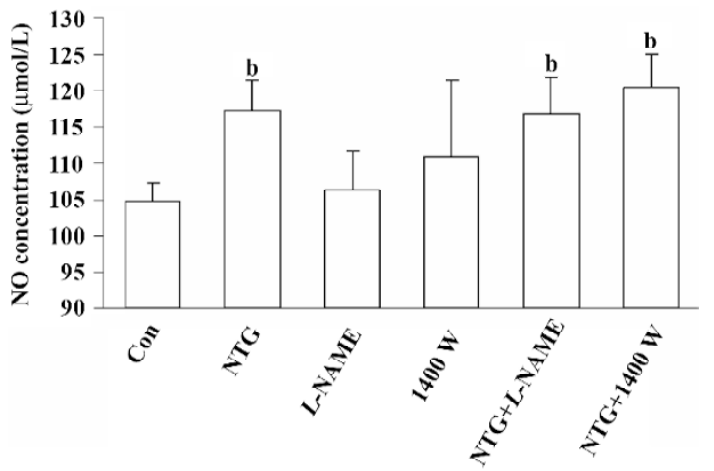

Figure 3. Effect of NOS inhibitors on NTG-mediated NO production in HBMSC cultures. HBMSC were treated with NTG $(10 \mu \mathrm{mol} / \mathrm{L})$ in the presence or absence of $L$-NAME $(300 \mu \mathrm{mol} / \mathrm{L})$ or $1400 \mathrm{~W}(100$ $\mu \mathrm{mol} / \mathrm{L})$. NO production was significantly increased in the NTGtreated group; however, there was no significant difference observed in NO production between the NTG-treated group and NTG $+L-\mathrm{NAME}$ or $1400 \mathrm{~W}$ groups, respectively. $n=3$. Mean \pm SD. ${ }^{\mathrm{b}} P<0.05$ vs vehicle control.

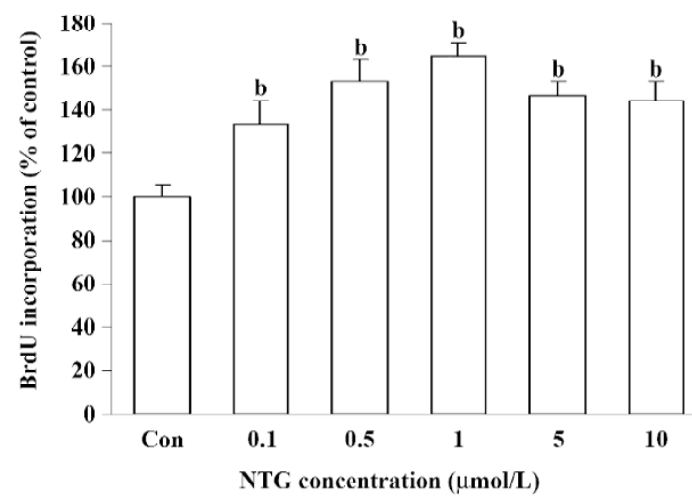

Figure 4. Effect of NTG on cell proliferation of HBMSC. BrdU OD $(\lambda / \mathrm{nm}=450)$ value was measured and expressed as the percentage of the control. NTG $(0.1-10 \mu \mathrm{mol} / \mathrm{L})$ dose dependently enhanced the cell proliferation of HBMSC and showed significant increase in BrdU incorporation. Maximal response of NTG was $1 \mu \mathrm{mol} / \mathrm{L} . \quad n=3$. Mean \pm SD. ${ }^{\mathrm{b}} P<0.05$ vs vehicle control.

present study, we measured ALP activity and the content of calcium deposition on $\mathrm{d} 8$ and 12 , respectively. The similar dose-dependent responses were observed in both ALP activity and calcium deposition (Figure 5A,6A). Consistent with the NO outcome in the conditioned media, $10 \mu \mathrm{mol} / \mathrm{L}$ NTG was found to exert maximal effect on osteoblastic differentiation in HBMSC cultures. In addition, there was a significant correlation between the NO production of NTG and the cellular ALP activity (Figure 5B), as well as the calcium deposition (Figure 6B), which indicated that the NO production of NTG was in line with the promotion of osteoblastic differentiation in HBMSC cultures. 
A

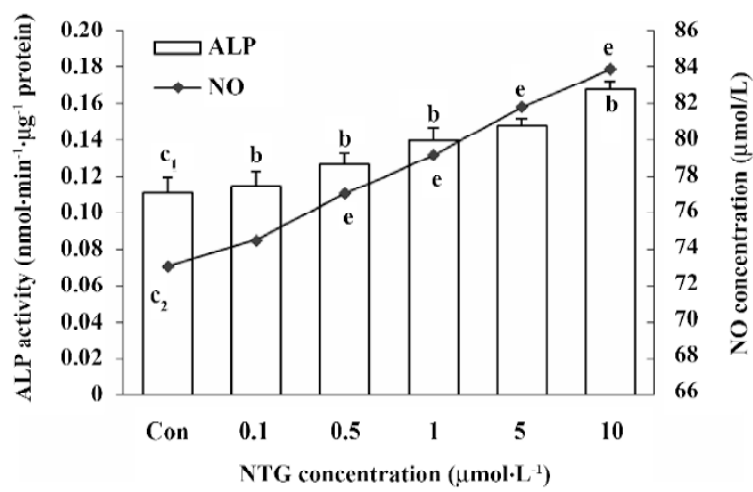

B

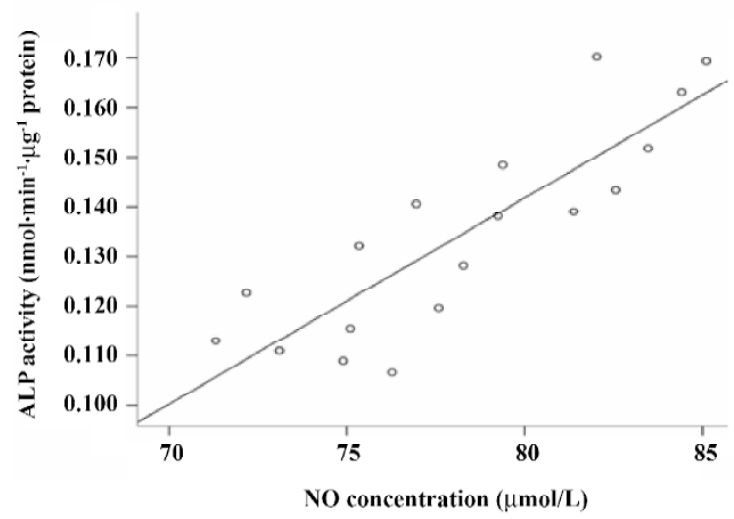

Figure 5. Effect of NTG on ALP activity of HBMSC. (A) ALP activity. NTG dose dependently enhanced the ALP activity in the early stage of osteoblastic differentiation of HBMSC cultures. Maximal effect of NTG was $10 \mu \mathrm{mol} / \mathrm{L}$, consistent with the maximal outcome of NO released in the conditioned media. $n=3$. Mean \pm SD. ${ }^{\mathrm{b}} P<0.05 v s \mathrm{c}_{1}$ vehicle control. ${ }^{\mathrm{e}} P<0.05 v s \mathrm{c}_{2}$ vehicle control. (B) Relationship between NO production and ALP activity. There was good correlation between NO production and ALP activity in the HBMSC cultures using two-tailed Pearson's correlation analysis; the correlation value was $r=0.856$.

Effect of NTG on RUNX2, osterix, and osteocalcin expressions of HBMSC cultures Real-time RT-PCR was performed to evaluate the effect of NTG on osteoblastic markers, such as RUNX2, osterix, and osteocalcin expressions on $\mathrm{d} 8$ of HBMSC cultures. As shown in Table 1, NTG (0.1-10 $\mu \mathrm{mol} / \mathrm{L})$ dose dependently promoted the osteoblast-specific transcriptional factor RUNX2 expression, with more than a 2-fold increase in $10 \mu \mathrm{mol} / \mathrm{L} \mathrm{NTG}$ treatment. Similarly, the RUNX2 downstream gene osteocalcin and another osteoblast-specific transcriptional factor osterix were also significantly induced in a dose-dependent manner. A slight but significantly decline of the gene expressions was also observed when treated with $300 \mu \mathrm{mol} / \mathrm{L} L$-NAME alone, indicating an
A

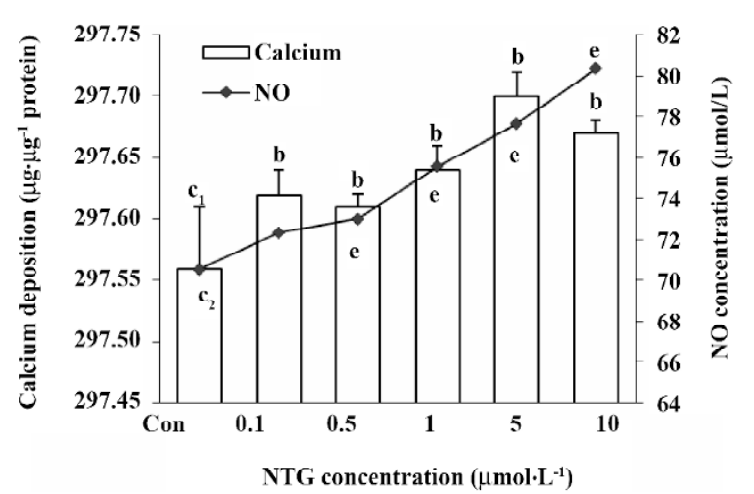

B

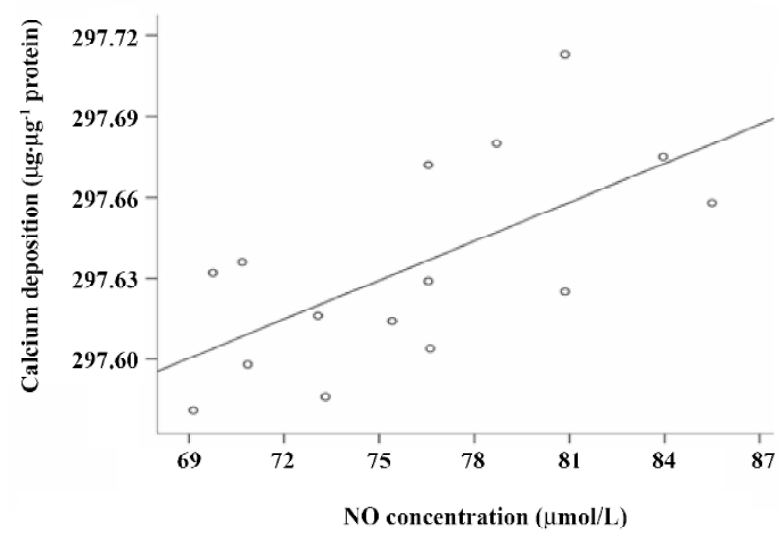

Figure 6. Effect of NTG on calcium deposition of HBMSC. (A) Calcium deposition. NTG dose dependently increased the calcium deposition in the latter stage of osteoblastic differentiation of HBMSC cultures. Similar to ALP activity, the maximal effect of NTG was 5 $\mu \mathrm{mol} / \mathrm{L} . \quad n=3$. Mean \pm SD. ${ }^{\mathrm{b}} P<0.05 v s \mathrm{c}_{1}$ vehicle control. ${ }^{\mathrm{e}} P<0.05 v s$ $\mathrm{c}_{2}$ vehicle control. (B) Relationship between NO production and calcium deposition. There was a significant correlation between NO production and calcium deposition in HBMSC cultures using two-tailed Pearson's correlation analysis; the correlation value was $r=0.648$.

Table 1. Effects of NTG on RUNX2, osterix, and osteocalcin expressions of HBMSC by real-time PCR. $n=4$. Mean \pm SD. ${ }^{b} P<0.05$, ${ }^{\mathrm{c}} P<0.01$ vs control $\left(\mathrm{Me}_{2} \mathrm{SO}\right) .{ }^{\mathrm{f}} P<0.01$ vs $L$-NAME.

\begin{tabular}{lccc}
\hline \multicolumn{1}{c}{ Group } & RUNX2 & Osteocalcin & Osterix \\
\hline Control & $1.00 \pm 0.12$ & $1.00 \pm 0.18$ & $1.00 \pm 0.25$ \\
NTG $(\mu \mathrm{mol} / \mathrm{L})$ & & & \\
0.1 & $1.35 \pm 0.13^{\mathrm{b}}$ & $1.48 \pm 0.16^{\mathrm{b}}$ & $1.10 \pm 0.13$ \\
1.0 & $1.62 \pm 0.15^{\mathrm{b}}$ & $2.01 \pm 0.22^{\mathrm{c}}$ & $1.63 \pm 0.24^{\mathrm{b}}$ \\
10 & $2.03 \pm 0.24^{\mathrm{c}}$ & $2.92 \pm 0.32^{\mathrm{c}}$ & $1.95 \pm 0.28^{\mathrm{c}}$ \\
$L-\mathrm{NAME}$ & $0.74 \pm 0.17^{\mathrm{b}}$ & $0.76 \pm 0.21^{\mathrm{b}}$ & $0.77 \pm 0.1^{\mathrm{b}}$ \\
NTG+L-NAME & $2.20 \pm 0.26^{\mathrm{f}}$ & $2.84 \pm 0.29^{\mathrm{f}}$ & $1.97 \pm 0.23^{\mathrm{f}}$ \\
\hline
\end{tabular}


inhibition of $L$-NAME on endogenous NOS activity and osteoblastic differentiation. However, this concentration of $L$-NAME could not block $10 \mu \mathrm{mol} / \mathrm{L}$ NTG-induced osteoblastic gene expression, suggesting that the NTG-induced osteoblastic differentiation is independent of endogenous NOS activity.

\section{Discussion}

In our previous study, we found that $17-\beta$-estradiol $\left(\mathrm{E}_{2}\right)$ and phytoestrogens, such as genistein ${ }^{[11]}$ and resveratrol ${ }^{[13]}$ had $\mathrm{E}_{2}$-like activity and enhanced cell proliferation and osteoblastic differentiation through the stimulation of NO production in mouse BMSC cultures. In the present study, we extended our observations to examine the effect of NTG, an NO donor, on cell proliferation and osteoblastic differentiation in human bone marrow cultures. Our data showed that NTG dose dependently elevated NO production in HBMSC cultures, which was independent of NOS activity. The release of NO by NTG also resulted in increased cell proliferation and osteoblastic differentiation of HBMSC, as evidenced by the increment of the BrdU incorporation, the induction of ALP activity in the early stage and the calcium deposition in the latter stage, and upregulation of osteoblastic markers, such as RUNX2, osteocalcin, and osterix expressions. These findings demonstrated that NTG stimulated cell proliferation and osteoblastic differentiation of HBMSC in vitro through a NO pathway.

NTG stimulates bone formation through the release of NO. NTG has been reported to prevent ovariectomy-induced bone loss in female rats ${ }^{[4]}$ and corticosteroid-induced bone loss in male rats ${ }^{[14]}$, but the mechanism remains unclear. According to the literature, the combination of $\mathrm{E}_{2}$ and NTG did not further enhance bone mass ${ }^{[15]}$, and reductions in the exercise-induced mRNA expression of human skeletal muscles by $L$-NAME can be prevented by the administration of $\mathrm{NTG}^{[16]}$, indicating that NTG prevents bone loss through the release of NO. In this regard, we found that NTG dose dependently elevated NO production, which is parallel with the induction of HBMSC cell proliferation and osteoblastic differentiation in vitro, supporting the view that NTG stimulates osteoblastic differentiation and bone formation through the release of NO. Consistent with our results, NTG has also been reported to counteract oophorectomy-induced bone loss in women as efficacious as standard estrogen replacement therapy ${ }^{[5]}$, and a significant decrease in the serum concentrations of nitrite/nitrate has also been noted in osteoporotic women compared to women with normal bone mineral density (BMD) or osteopenia ${ }^{[17]}$. In addition, we found that the NO release of NTG was independent of cellular NOS activity with high dosages of NTG, which was inconsistent with the in vivo rat model previously reported ${ }^{[18]}$. This could be due to gene regulations in systemic conditions. It was also observed in our study that the reduction of bone marker gene expressions caused by $L$-NAME was reversed by NTG, which further indicated that the effect of NTG was not completely dependent on NOS. Jamal et $a l^{[19]}$ reported that the intermittent use of nitrates has been shown to enhance BMD and decrease the risk of fractures in osteoporotic women, further supporting the view that an increase of NO production can treat human osteoporosis in future.

NTG exerted its anabolic effects through either NO/cGMP or cGMP-independent pathways. NO was reported to act through the cGMP-independent pathway by nitrifying the functional group in proteins ${ }^{[20]}$ and reacting with superoxide anion $^{[21]}$. It has been shown that SNAP, another NO donor, facilitated the proliferation of MC3T3-E1 through NO-COX-PGE loop, independent of the cGMP pathway ${ }^{[22]}$. This could also be an example of NTG enhancing the proliferation of human BMSC cultures. Two types of guanylate cyclase (GC) have been reported to express in osteoblastic precursor cells ${ }^{[1,23]}$, indicating that the NO/GC/cGMP pathway may play an important role in NTG-induced cell proliferation and osteoblastic differentiation of HBMSC cultures. Other studies have shown that a slow and moderate release of NO stimulates the replication of primary rat osteoblasts and ALP activity via the cGMP-dependent pathway ${ }^{[24]}$, and c-fos, one of the genes controlling cell proliferation, could be upregulated by the cellular cGMP level ${ }^{[25]}$. Furthermore, our recent research found that $E_{2}$ and genistein enhanced cell proliferation and osteoblastic differentiation of mouse BMSC via the NO/cGMP pathway, and that genistein-induced osteoblastic makers, such as RUNX2 (Cbfa1) and osteocalcin expressions were markedly attenuated by using ODQ, an inhibitor of sGC, indicating that the upregulation of osteoblastic differentiation gene transcription in BMSC cultures are dependent on cellular cGMP levels ${ }^{[11]}$. Consistent with these findings and our current data about NTG upregulating osteoblastic markers, such as RUNX2 expression, EDA-NO, a NO donor, was reported to directly phosphorylate RUNX2 (Cbfa1) via the cGMP/PKG pathway to increase its downstream gene MMP13 expression $^{[26]}$. Moreover, the administration of potassium nitrosylpentachlorouthenate, another NO donor, reversed the decline of the osteoblast number and increased the expressions of RUNX2 (Cbfa1) and osteocalcin genes in eNOS knockout mice after 21 days treatment ${ }^{[27]}$. Taken together, the NO/cGMP/RUNX2 (Cbfa1) pathway plays an essential in NTG-mediated cell proliferation and osteoblastic differentiation of mouse and human BMSC cultures ${ }^{[28,29]}$. 
In conclusion, the present study is the first to confirm that NTG, through the direct release of NO, stimulates cell proliferation and osteoblastic differentiation in HBMSC cultures. The activation of the NO pathway may mediate the anabolic effects of NTG on bone cells and serve as an important mechanism for this common NO donor effectiveness as an anti-osteoporotic treatment.

\section{References}

1 Collaborative Group on Hormone Factors in Breast Cancer. Breast cancer and hormone replacement therapy: collaborative reanalysis of data from 51 epidemiological studies of 52705 women with breast cancer and 108411 women without breast cancer. Lancet 1997; 350: 1047-59.

2 Qu Q, Perälä-Heape M, Kapanen A, Dahllund J, Salo J, Väänänen $\mathrm{HK}$, et al. Estrogen enhances differentiation of osteoblasts in mouse bone marrow culture. Bone 1998; 400: 201-9.

3 Aguirre J, Buttery L, O'Shaughnessy M, Afzal F, Fernandez de Marticorena I, Hukkanen M, et al. Endothelial nitric oxide synthase gene-deficient mice demonstrate marked retardation in postnatal bone formation, reduced bone volume, and deficient in osteoblasts maturation and activity. Am J Pathol 2001; 158: 247-57.

4 Hukkanen M, Platts LA, Lawes T, Girgis SI, Konttinen YT, Goodship AE, et al. Effect of nitric oxide donor nitroglycerin on bone mineral density in a rat model of estrogen deficiency-induced osteopenia. Bone 2003; 32: 1-8.

5 Wimalawansa SJ. Nitroglycerin therapy is as efficacious as standard estrogen replacement therapy (Premarin) in prevention of oophorectomy-induced bone loss: a human pilot clinical study. J Bone Miner Res 2000; 15: 2240-4.

6 Jiang X, Gittens SA, Chang Q, Zhang X, Chen C, Zhang Z. The use of tissue-engineered bone with human bone morphogenetic protein-4-modified bone-marrow stromal cells in repairing mandibular defects in rabbits. Int J Oral Maxillofac Surg 2006; 35 : 1133-9.

7 Mankani MH, Kuznetsov SA, Wolfe RM, Marshall GW, Robey PG. In vivo bone formation by human bone marrow stromal cells: reconstruction of the mouse calvarium and mandible. Stem Cells 2006; 24: 2140-9.

8 Dai ZJ, Li YL, Quarles LD, Xiao ZS. Resveratrol enhances proliferation and osteoblastic differentiation in human mesenchymal stem cells via ER-dependent ERK1/2 activation. Phytomedicine 2007; 14: 806-14.

9 Xiao ZS, Simpson LG, Quarles LD. IRES-dependent translational control of Cbfa1/Runx2 expression. J Cell Biochem 2003; 88: 493-505.

10 Wang FH, Jin WF, Gao JJ. The evaluation of pharmacodynamic action to drugs for osteoporosis. Chin J Osteoporosis 1999; 5: 58-62.

11 Pan W, Quarles LD, Xiao ZS. Genistein stimulates the osteoblastic differentiation via NO/cGMP in bone marrow culture. J Cell Biochem 2005; 94: 307-16.

12 Xiao ZS, Quarles LD, Chen QQ, Yu YH, Qu XP, Jiang CH, et al.
Effect of asymmetric dimethylarginine on osteoblastic differentiation. Kidney Int 2001; 60: 1699-704.

13 Song LH, Pan W, Yu YH, Quarles LD, Zhou HH, Xiao ZS. Resveratrol prevents CsA inhibition of proliferation and osteoblastic differentiation of mouse bone marrow-derived mesenchymal stem cells through an ER/NO/cGMP pathway. Toxicol In Vitro 2006; 20: 915-22.

14 Wimalawansa SJ, Chapa MT, Yallampalli C, Zhang R, Simmons DJ. Prevention of corticosteroid-induced bone loss with nitric oxide donor nitroglycerin in male rats. Bone 1997; 21: 275-80.

15 Wimalawansa SJ, De Marco G, Gangula P, Yallampalli C. Nitric oxide donor alleviates ovariectomy-induced bone loss. Bone 1996; 18: 301-4.

16 Steensberg A, Keller C, Hillig T, Frøsig C, Wojtaszewski JF, Pedersen BK, et al. Nitric oxide production is a proximal signaling event controlling exercise-induced mRNA expression in human skeletal muscle. FASEB 2007; 21: 2683-94.

17 Sanada M, Taguchi A, Higashi Y, Tsuda M, Kodama I, Yoshizumi $\mathrm{M}$, et al. Forearm endothelial function and bone mineral loss in postmenopausal women. Atherosclerosis 2004; 176: 387-92.

18 He MH, Wang ZL, Wu J, Chen T, Pang W, Yang XM. Changes of serum nitric oxide and nitric oxide synthase in ovariectomy rats model of osteoporosis after nitroglycerin therapy. Chin J Endemiol 2004; 23: 438-40.

19 Jamal SA, Browner WS, Bauer DC, Cummings SR. Intermittent use of nitrates increases bone mineral density: the study of osteoporotic fractures. J Bone Min Res 1998; 13: 1755-9.

20 Lander HM, Ogiste JS, Pearce SF, Levi R. Nitric oxide-stimulated guanine nucleotide exchange on p21ras. J Biol Chem 1995; 270: 7017-20.

21 Feelisch M, Stamler JS. Methods in nitric oxide research. Chichester: Wiley \& Sons 1996.

22 Kanamaru Y, Takada T, Saura R, Mizuno K. Effect of nitric oxide on mouse clonal osteogenic cell, MC3T3-E1, proliferation in vitro. Kobe J Med Sci 2001; 47: 1-11.

23 Inoue A, Hiruma Y, Hirose S, Yamaguchi A, Hagiwara H. Reciprocal regulation by cyclic nucleotides of the differentiation of rat osteoblast-like cells and mineralization of nodules. Biochem Biophys Res Commun 1995; 215: 1104-10.

24 Mancini L, Moradi-Bidhendi N, Becherini L, Martineti V, MacIntyre I. The biphasic effects of nitric oxide in primary rat osteoblasts are cGMP dependent. Biochem Biophys Res Commun 2000; 274: 477-81.

25 Pilz RB, Casteel DE. Regulation of gene expression by cyclic GMP. Circ Res 2003; 93: 1034-46.

26 Zaragoza C, López-Rivera E, García-Rama C, Saura M, MartínezRuíz A, Lizarbe TR, et al. Cbfa-1 mediates nitric oxide regulation of MMP-13 in osteoblasts. J Cell Sci 2006; 119: 1896-902.

27 Ducy P, Zhang R, Geoffroy V, Ridall AL, Karsenty G. Osf2/ Cbf $\alpha 1$ : a transcriptional activator of osteoblast differentiation. Cell 1997; 89: 747-54.

28 Afzal F, Polak J, Buttery L. Endothelial nitric oxide synthase in the control of osteoblastic mineralizing activity and bone integrity. J Pathol 2004; 202: 503-10.

29 Xiao ZS, Zhou HH. Progress in Cbf $\alpha 1$ gene and its regulation. Chin Pharmacol Bull 2001; 17: 365-8. 